\title{
El artesano de La Canal dels Avellaners (Edad de Bronce, Barcelona): análisis de huellas dactilares
}

\author{
The artisan of La Canal dels Avellaners (Bronze Age, Barcelona): analysis of fingerprints
}

\author{
Virginia Míguez ${ }^{\mathrm{a}}$, Pere Ibáñez-Gimeno ${ }^{\mathrm{ab}}$, Josep Carreras ${ }^{\mathrm{c}}$, Josep Liria ${ }^{\text {ac }}$ y Assumpció Malgosa ${ }^{\mathrm{a}}$
}

\section{RESUMEN}

Trabajos recientes han descrito la existencia de diferencias sexuales y de edad en la anchura de las crestas epidérmicas en huellas dactilares humanas. El potencial forense de esta característica es muy amplio y se ha empezado a utilizar para obtener información a partir de las huellas encontradas tanto en el lugar del crimen como en objetos cerámicos antiguos. El objetivo de este estudio es determinar el sexo y la edad aproximada del autor de los dermatoglifos encontrados en un fragmento cerámico de La Canal dels Avellaners, Berga, Cataluña. Las huellas fueron comparadas con una muestra de población española contemporánea que incluía individuos de entre 6 y 58 años. Los resultados indican que el artesano fue un hombre adulto y demuestran que este tipo de investigaciones informan sobre la persona que las dejó y, por tanto, presenta un gran potencial en estudios sobre aspectos sociales de la elaboración de cerámicas en culturas antiguas. laners, Berga, Catalonia. The ancient fingerprints were compared with a sample from the Spanish contemporary population, including individuals from 6 to 58 years of age. The results indicate that the owner of these fingerprints was an adult male. This research shows that the study of ancient fingerprints can provide important information about the person who left them and it has a great potential to determine the social background of ceramicsmaking in ancient cultures.

Palabras clave: Arqueología forense; Paleodermatoglifos; Dermatoglifos; Anchura de la cresta epidérmica; Cerámicas prehistóricas; Diferencias sexuales; Diferencias de edad.

Keywords: Forensic archaeology; Ancient fingerprints; Fingerprints; Epidermal ridge breadth; prehistoric ceramics; Sexual differences; Age differences.

\begin{abstract}
Several studies have reported sex and age differences in epidermal ridge breadth in human fingerprints and analyzed its variability in different populations. In recent years, some investigations have explored the forensic application of this feature for inferring information from fingerprints found at a crime scene or on ancient ceramic artifacts. The objective of this study is to determine the sex and approximate age of the owner of the fingerprints found in a ceramic fragment from La Canal dels Avel-
\end{abstract}

\section{INTRODUCCIÓN}

Los dermatoglifos son las impresiones de las crestas epidérmicas o crestas papilares y de los surcos interpapilares de dedos (huellas dactilares), manos (huellas palmares) y pies (huellas plantares) que se generan cuando cualquiera de ellos entran en contacto con otro objeto. Como resultado de ese contacto, la morfología de las

a Unitat d'Antropologia Biològica, Dept. de Biologia Animal, Biologia Vegetal i Ecologia. Universitat Autònoma de Barcelona. 08193 Bellaterra. Barcelona. España. Correos e.: virginia.miguez.m@gmail.com; assumpcio.malgosa@uab.cat

b PAVE Research Group and McDonald Institute for Archaeological Research, Dept. of Archaeology and Anthropology. University of Cambridge. Pembroke street. Cambridge CB2 3DX. Reino Unido. Correo e.: pereibgi@gmail.com

c Societat d'Arqueologia del Berguedà. Carrer de la Justícia 7. 08600 Berga. Barcelona. España.

Correos e.: josepcarrerasbalague@gmail.com; 18442jlc@comb.cat;

Recibido: 15-X-2015; aceptado: 3-III-2016. 
crestas epidérmicas queda latente o grabada en la superficie del objeto. Son una herramienta clave en las investigaciones criminales, ya que permiten la identificación individual.

Los dermatoglifos son características poligénicas con una posible influencia ambiental en los primeros meses de vida intrauterina (Holt 1968; Loesch 1983). Una vez formadas y en ausencia de lesiones profundas de la dermis, las crestas que forman las huellas permanecen invariables durante la vida del individuo. Se han realizado numerosos estudios sobre la diversidad de los dermatoglifos en distintas poblaciones humanas, pero la mayoría se han basado en características del dibujo o del trayecto y dimensiones de la cresta. Otras características interesantes como la anchura de las crestas se han investigado con menos asiduidad. En los últimos años, varios equipos de investigación se han interesado, precisamente, en aspectos como la densidad de la cresta, con el objetivo de inferir el sexo de los individuos en la población española (Gutiérrez-Redomero et al. 2008), la población americana de ascendencia europea y africana (Acree 1999), la egipcia (Eshak et al. 2012), la india (Nayak et al. 2010a), la china y malaya (Nayak et al. 2010b), la argentina (Rivaldería et al. 2016), la filipina (Taduran et al. 2015) o la del grupo indígena amerindio Mataco-Mataguayo (Gutiérrez-Redomero et al. 2011). Estas investigaciones han puesto de manifiesto diferencias en la densidad de las crestas (y, por tanto, en su anchura) entre sexos (Ohler y Cummins 1942; Mundorff et al. 2014), diferencia entre dedos y entre zonas de un mismo dedo (Cummins et al. 1941). Otros estudios han explorado las diferencias entre poblaciones (Gutiérrez-Redomero et al. 2013a, b) y las existentes entre individuos de distintas edades (David 1981). Los patrones de las huellas no cambian durante la vida, pero la distancia entre las crestas aumenta con el tiempo, incrementándose durante la infancia y estabilizándose en la edad adulta. Algunas investigaciones incluso sugieren que ciertas edades presentan una anchura estándar entre crestas (David 1981; Kamp et al. 1999).

Teniendo en cuenta la gran cantidad de información que se puede recuperar de los dermatoglifos, los estudios de huellas sobre objetos antiguos (paleodermatoglifos) han comenzado a tomar relevancia, siendo diversos los materiales que pueden conservarlas. En concreto la cerámica es un material ideal para la producción y preservación de dermatoglifos. Es suficientemente plástica para la impresión y tras la cocción se transforma en un material duro y estable que conserva las huellas durante largos periodo de tiempo. Además, cualquier pieza de cerámica manufacturada ha estado en contacto directo con manos humanas y esto la convierte en un medio potencial de transferencia.

Cummins (1941) dividió las huellas en cerámica en dos grupos: (1) intencionadas (firmas o diseños decorativos y con significado) y (2) accidentales, resultado fortuito del modelaje. $\mathrm{La}$ mayoría de las huellas halladas son accidentales y, por tanto, pequeñas y fragmentarias. Su presencia en la cerámica prehistórica no es muy común, ya sea porque pasan desapercibidas al no observarse los fragmentos meticulosamente o porque el lavado riguroso de los mismos, muchos de cocción defectuosa, impide identificar los dermatoglifos.

Las huellas en cerámica son el negativo de las crestas epidérmicas humanas. Sin un patrón dermatoglífico aparente no existe evidencia cierta de que una marca en la cerámica sea una huella dactilar. No obstante algunos marcadores (anchura de la cresta estable, presencia de minucias y rugosidad a lo largo de las crestas), bajo condiciones concretas, pueden identificar incluso las huellas más pequeñas (Králík et al. 2002). El estudio de las huellas antiguas en objetos de cerámica ha permitido determinar, en algunos casos, la edad de las personas que participaron en su creación (Kamp et al. 1999; Jägerbrand 2007).

La anchura media de la cresta epidérmica (Mean Ridge Breath, MRB) observada en cerámicas se puede utilizar como un indicador de edad (desde el nacimiento hasta la madurez) y del sexo (en edad adulta) del posible fabricante del artefacto (Králík y Novotný 2003). Algunos investigadores han probado que los niños participaban en la producción de objetos de cerámica durante la Edad de Bronce (Jägerbrand 2007; Alarcón García 2015).

El objetivo de este trabajo es determinar la edad aproximada y el sexo del fabricante de una cerámica con dermatoglifos encontrada en $\mathrm{La} \mathrm{Ca}$ nal dels Avellaners (Sierra de Queralt, El Berguedà, Catalunya) mediante el estudio de la anchura de las crestas y su comparación con una muestra de individuos de la población española actual. 


\section{MATERIALES Y MÉTODOS}

\subsection{Material}

La cavidad rocosa llamada La Canal dels Avellaners (Berga, El Berguedà, Cataluña) (Fig. 1) contiene restos arqueológicos de varias culturas como el fragmento cerámico en estudio. Se abre a una altura de 995 m s.n.m. en la Sierra de Queralt formada por un anticlinal con materiales calcáreos del Cretácico, Oligoceno y Eoceno. El desprendimiento de una gran roca de la mole calcárea dejó habitable la pequeña superficie de $22 \mathrm{~m}^{2}$ donde se localiza el yacimiento. La propia configuración rocosa la resguarda de lluvias y vientos y actúa como una chimenea natural cuando se hace fuego en su interior. Estas características motivaron una ocupación casi sin interrupción desde el Neolítico medio hasta la época medieval. La cavidad está actualmente oculta por una exuberante vegetación. El yacimiento tenía un potencial de sedimentos de $5,20 \mathrm{~m}$ repartido en 16 estratos, 12 de ellos fértiles y 4 estériles.

El estrato fértil VII nivel J, situado a 2,18 m de profundidad y con una potencia de $65 \mathrm{~cm}$, y correspondiente a la Edad del Bronce contenía el fragmento con dermatoglifos. Dicho fragmento se encontró en la parte inferior del estrato, junto a restos de vasos con asa de botón y otros restos de jarra con decoración ungular en toda la superficie de la pieza, muy típica de la Edad del Bronce medio, c.1500 años aC. (Carreras 1990).

El fragmento pertenece a una jarra con borde exvasado y un pezón de prensión a $6 \mathrm{~cm}$ del borde. Bajo el borde se identificaron los dermatoglifos (Fig. 2). Unos tres centímetros por debajo

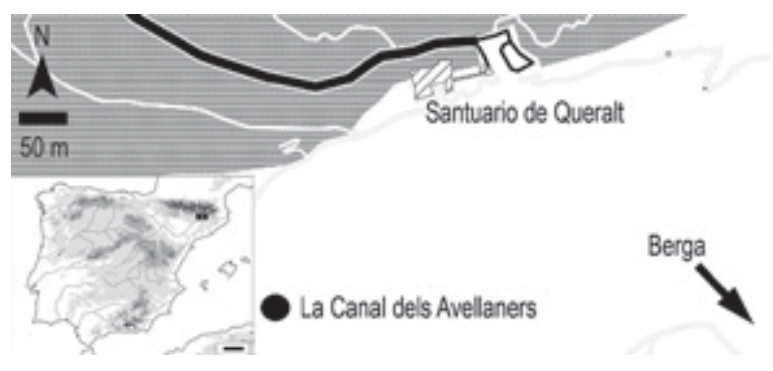

Fig. 1. Localización de la cavidad La Canal dels Avellaners en la Sierra de Queralt, en el noreste de la Península Ibérica. Mapa extraído de maps.stamen.com. La escala en el mapa inferior izquierdo es de $100 \mathrm{~km}$.

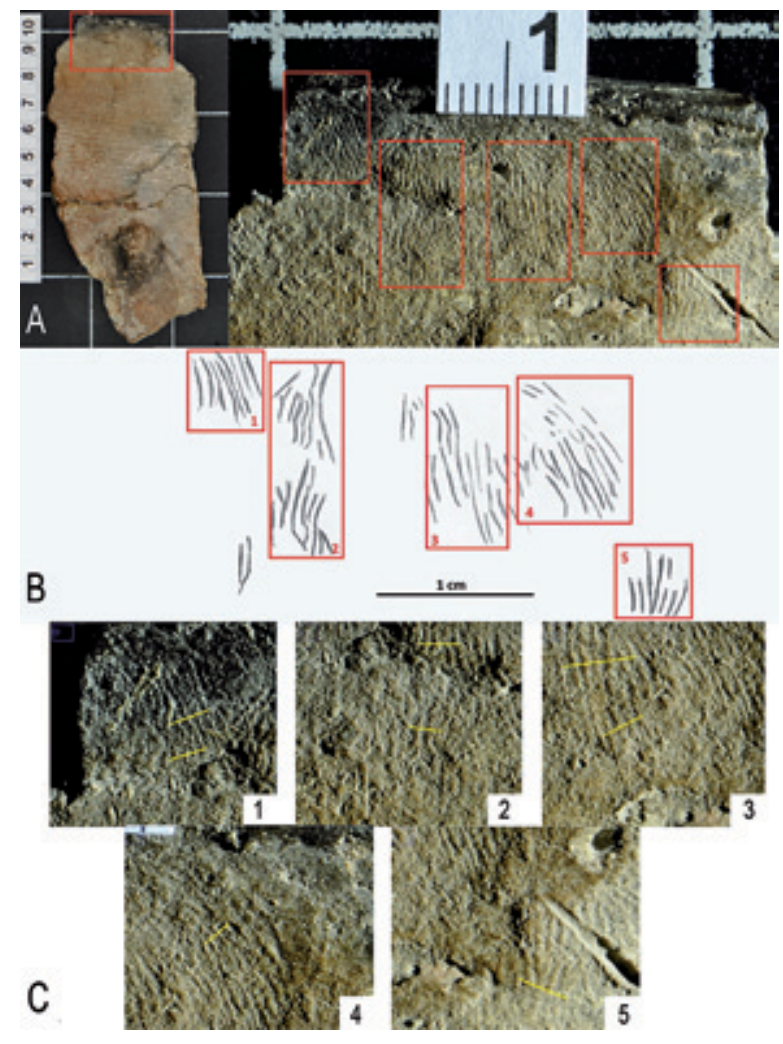

Fig. 2. A. Fragmento de la jarra cerámica de La Canal dels Avellaners, decorada posiblemente a peine, con huellas dactilares (izquierda) y subdivisión en 5 regiones (derecha). B. Esquema de las huellas a partir del cual se han determinado las regiones. C. Imagen de las medidas tomadas en las 5 regiones.

empieza una decoración de pequeños surcos horizontales, posiblemente a peine. La pasta de arcilla contiene un desgrasante muy fino, no muy habitual en cerámicas prehistóricas que suelen contener piedrecitas de hasta $5 \mathrm{~mm}$ (Carreras 1990).

\subsection{Métodos}

\subsubsection{Descriptivos}

El valor arqueológico de la pieza cerámica ha exigido su estudio mediante métodos no invasivos. Las huellas dactilares en cerámicas se detectan gracias al contraste entre luces y sombras en un negativo tridimensional. En este caso se han analizado mediante macrofotografía (Nikon D700 
con lente macro) con iluminación halógena de incidencia oblicua. Se han observado y medido 5 huellas.

Penrose (1968: p. 1) definió la anchura real de la cresta como "la distancia entre el centro de un surco epidérmico y el centro del siguiente surco, definiendo una línea que forma un ángulo recto con la dirección del surco". La anchura real es indeterminable por métodos directos, por lo que se han utilizado los indirectos. En las huellas en objetos antiguos, Králík y Novotoný (2003) definieron la anchura de la cresta como la distancia perpendicular entre el margen de una sombra y el mismo punto en el margen de la siguiente (Fig. 3). La cocción y secado contraen la cerámica y pueden reducir la anchura de las crestas hasta un 20\% sin que sea posible su cuantificación exacta. En este estudio, la corrección ha sido del 7,5\% por ser el valor que ha estimado mejor la anchura de la cresta en estudios previos ${ }^{1}$.

El sexo y la edad aproximada del individuo al que pertenecían los paleodermatoglifos de la cerámica se han determinado por comparación con la población actual. Se han recogido las huellas

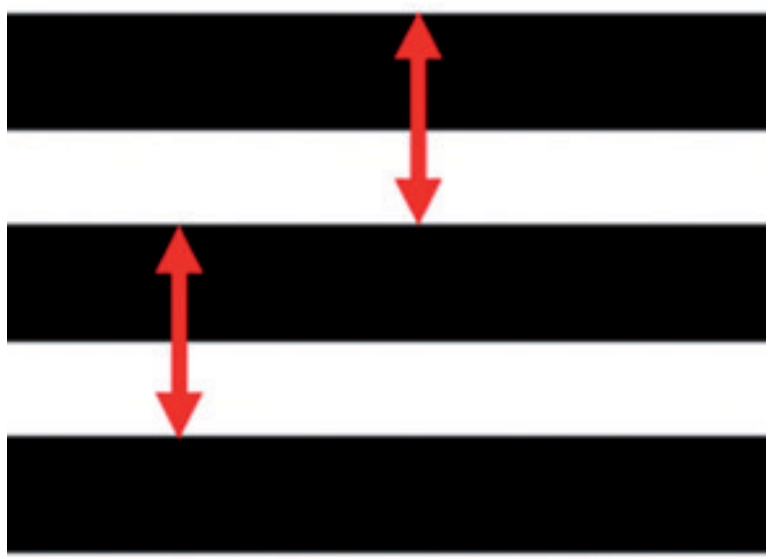

Fig. 3. Anchura de la cresta en huellas encontradas en cerámicas y otros objetos antiguos. Adaptada de Králík y Novotoný 2003: p. 8.

\footnotetext{
${ }^{1}$ Králík, M. 2000: Otisky prstů a dlaní na keramick ém materiálu (Fingerprints and palm-prints on ceramics). Tesis de Máster. Department of Anthropology, Faculty of Science, Masaryk University Brno.
}

\begin{tabular}{|l|l|c|c|}
\hline \multicolumn{2}{|c|}{$\leq 25$ años } & Masculinos & Femeninos \\
\cline { 2 - 4 } & Media & 13,10 & 14,12 \\
\cline { 2 - 4 } & Desviación estándar & 4,52 & 5,20 \\
\cline { 2 - 4 } & Mínimo & 6 & 8 \\
\cline { 2 - 4 } & Máximo & 25 & 24 \\
\cline { 2 - 4 } & $\mathrm{N}$ & 89 & 102 \\
\hline > 25 años & Media & 36,60 & 41,12 \\
\cline { 2 - 4 } & Desviación estándar & 11,82 & 16,50 \\
\cline { 2 - 4 } & Mínimo & 26 & 26 \\
\cline { 2 - 4 } & Máximo & 57 & 86 \\
\cline { 2 - 4 } & $\mathrm{N}$ & 15 & 17 \\
\hline
\end{tabular}

Tab. 1. Estadísticos descriptivos para la edad (en años) de los individuos de la muestra de población actual.

de una muestra de 222 individuos de padres y abuelos españoles de entre 6 y 58 años, compuesta por un número semejante de hombres (104) y mujeres (118) tanto en individuos adultos $(>25$ años) como en individuos jóvenes e infantiles ( $\leq 25$ años) (Tab. 1).

Existe una correlación significativa entre el desarrollo de las crestas epidérmicas y el tamaño de la palma, así como entre la anchura del espacio entre crestas y el tamaño del esqueleto en general, el grueso dérmico alrededor de la falange distal y la longitud de los metacarpos (Babler 1990; Loesch y Lafranchi 1990). La anchura de las crestas aumenta durante el crecimiento hasta alcanzar un máximo en la edad adulta, cuando deja de variar (David 1981; Kamp et al. 1999). Por ello todos los individuos mayores de 25 años se han considerado en conjunto.

Las huellas de la población actual se han obtenido mediante toma posada, impregnando la epidermis con grafito y recuperando el dibujo con un papel autoadhesivo (Aase y Lyons 1971). Todos los individuos participantes de la investigación han cedido voluntariamente las muestras utilizadas. Los consentimientos informados de los individuos menores de 18 años han sido firmados por sus padres o representantes legales.

La variabilidad intraindividual se ha evaluado recogiendo muestras de los dedos pulgar e índice, derecho e izquierdo. Al ser los más utilizados al manipular objetos (Almécija 2009) tienen más probabilidad de quedar grabados en

Trab. Prehist., 73, N. ${ }^{\circ}$ 1, enero-junio 2016, pp. 147-159, ISSN: 0082-5638

doi: $10.3989 /$ tp.2016.12168 
una cerámica durante su proceso de fabricación. Las muestras han sido escaneadas (EPSON Perfection 3170) para su posterior análisis con el programa Image J 1.45s (2011) con el cual se han medido las crestas. Como aproximadamente el 90\% de la población es diestra (Subirà y Malgosa 1988; McGrew y Marchant 1994; Perelle y

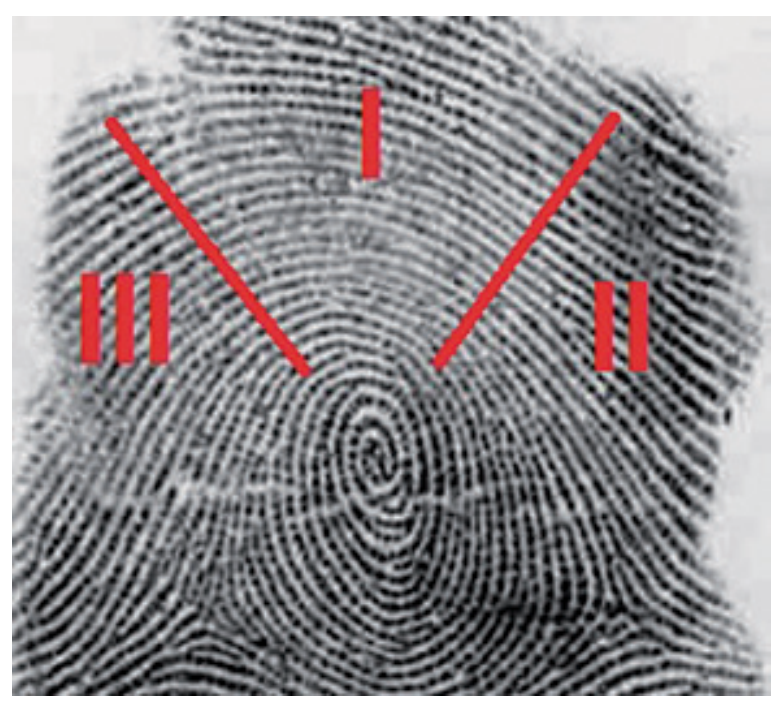

Fig. 4. División de la huella dactilar en tres zonas para tomar las medidas.
Ehrman 1994) y en la muestra no hay diferencias significativas entre la anchura de las crestas de los pulgares (Wilcoxon: $Z=-0$ '275; $N=217$; $\mathrm{p}>0$ '05) y de los índices (Wilcoxon: $Z=-1$ '095; $\mathrm{N}=206$; $\mathrm{p}>0$ ’05) de ambas manos, la comparación de las huellas en la cerámica se ha limitado a las muestras de los dedos pulgar e índice de la mano derecha.

Para medir la anchura de la cresta y, dada la variabilidad entre las regiones del dedo, se han definido tres zonas de análisis: distal (I), lateral (II) y medial (III) (Fig. 4). Como se buscaba obtener datos incuestionables se han usado solo las huellas claramente legibles. Ello ha llevado a desestimar algunas, sobre todo, de individuos infantiles, afectando al número final de datos considerados. Se han analizado un total de 616 medidas en pulgar (zonas I 209; II 212; III 195) y 570 medidas en índices (zonas I 187; II 194; III 189).

En las medidas sobre muestras de huellas en tinta o grafito, la anchura de la cresta se puede determinar trazando una línea transversal a un número concreto de crestas y dividiendo la medida obtenida por este número (Fig. 5). En este caso, la anchura mínima medida ha sido de 6 crestas, llegándose hasta las 10 siempre que fue posible.
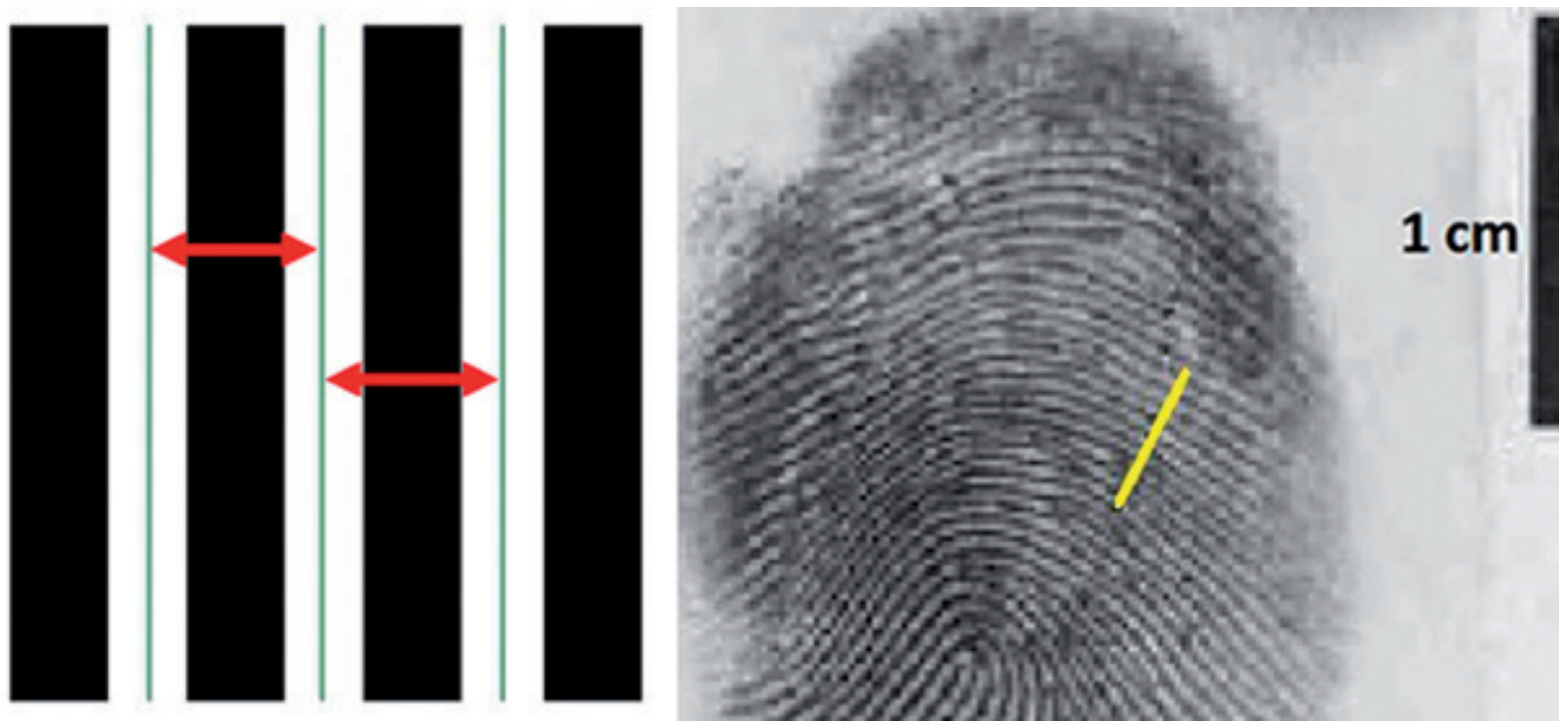

Fig. 5. Anchura de la cresta en huellas en tinta y grafito (izquierda) y ejemplo de medida (derecha). 


\subsubsection{Análisis estadísticos}

Los datos se han analizado mediante el test de Kolmogorov-Smirnov. Los resultados indican que no todas las variables presentan una distribución normal, por lo que se han manejado pruebas no paramétricas en los análisis estadísticos.

Las tres medidas de cada dedo se han comparado mediante el test de Friedman para muestras relacionadas. En los análisis posteriores, se han utilizado las medias de las medidas de cada dedo. Para la determinación de diferencias entre pulgar e índice se ha recurrido al test de Wilcoxon para muestras relacionadas. Para precisar la relación entre anchura de la cresta y edad del individuo, se han realizado regresiones logarítmicas. Por último, para testar las diferencias sexuales en los individuos adultos, se ha empleado el test de Mann-Whitney para muestras independientes.

Los resultados obtenidos a partir de la base de datos de la población de referencia se han comparado con las huellas encontradas en la cerámica para determinar la edad y el sexo del autor potencial. Primero se ha determinado la edad aproximada del individuo a partir de la regresión obtenida con los datos de la población actual. Seguidamente se han realizado análisis discriminantes para determinar el sexo. Todos los test estadísticos se han realizado con el programa IBM SPSS Statistics 21 (2012).

\section{RESULTADOS}

\subsection{Huellas de la cerámica}

Se han determinado un mínimo de 5 huellas diferentes y potencialmente medibles en la cerá- mica a partir de la direccionalidad y la separación entre ellas (Fig. 2A, B). Considerando la longitud y conservación de las huellas se han efectuado dos medidas de la anchura de las crestas para las regiones 1,2 y 3 y una medida para las regiones 4 y 5 (Fig. 2C). Para el análisis de las tres primeras, se han utilizado los valores medios de las dos medidas (Tab. 2).

\subsection{Población de referencia}

Los resultados obtenidos para toda la muestra indican que existen diferencias significativas entre las medidas de las diferentes zonas en el dedo pulgar (test de Friedman: $\mathrm{X}^{2}=6$ '668; $\mathrm{N}=185$; $\mathrm{p}<0$ '05) y en el dedo índice (test de Friedman: $X^{2}=6$ '215; $N=158 ; p<0$ '05), siendo las crestas de la zona I las mas anchas (Fig. 6). La media de la anchura de las tres zonas del dedo pulgar es significativamente mayor que la media de las del dedo índice (test de Wilcoxon: $Z=-2$ '984; $N=210$; $\mathrm{p}<0$ '005) (Fig. 6).

\subsection{Estimación de la edad del individuo}

El mejor modelo para analizar la relación entre edad y anchura de la cresta en la muestra de individuos de referencia ha sido la regresión logarítmica. Las regresiones son significativas para los dos sexos únicamente en los individuos de 25 años o menos. No se puede descartar que esta falta de asociación en los mayores de 25 años, en ambos dedos (Tab. 3; Fig. 7), esté en parte afectada por el tamaño de la muestra en este grupo. Según estos resultados se puede predecir la edad aproximada

\begin{tabular}{|l|c|c|c|c|c|}
\hline & Región 1 & Región 2 & Región 3 & Región 4 & Región 5 \\
\hline Medida 1 & 0,0535 & 0,0510 & 0,0638 & 0,0520 & 0,0524 \\
\hline Medida 2 & 0,0515 & 0,0543 & 0,0578 & & \\
\hline \multirow{2}{*}{ Corrección (7,5\%) } & 0,0575 & 0,0548 & 0,0686 & 0,0557 & 0,0563 \\
\hline Media región & 0,0553 & 0,0584 & 0,0621 & & 0,0563 \\
\hline
\end{tabular}

Tab. 2. Valores de la anchura media de la cresta epidérmica (Mean Ridge Breath) en las distintas regiones de la cerámica, valores corregidos del $M R B$ y media del $M R B$ corregido para cada región $(\mathrm{cm})$.

Trab. Prehist., 73, N. ${ }^{\circ}$ 1, enero-junio 2016, pp. 147-159, ISSN: 0082-5638

doi: $10.3989 /$ tp.2016.12168 

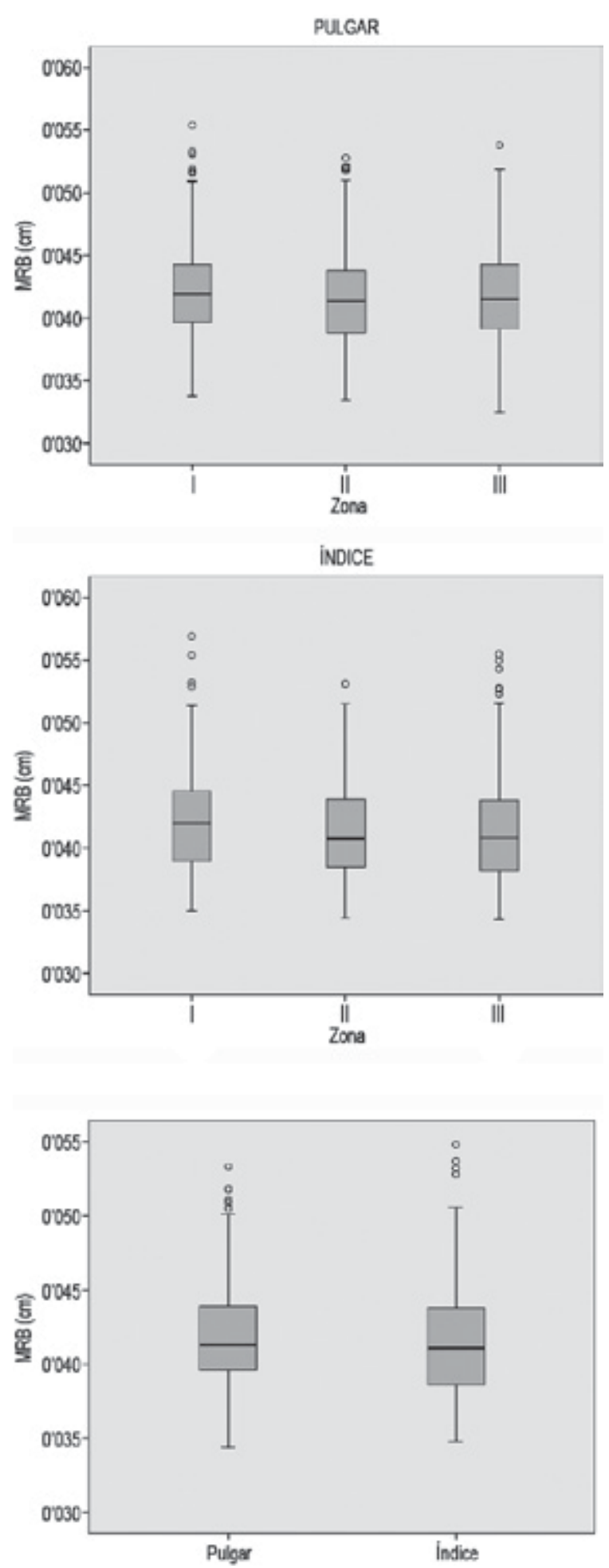

Fig. 6. Anchura media de la cresta epidérmica (Mean Ridge Breath, $M R B)$ de toda la población actual de referencia: arriba en las tres zonas de pulgar e índice; abajo, media en dichas zonas.

\begin{tabular}{|c|c|l|c|c|}
\hline & Edad & \multicolumn{1}{|c|}{ Sexo } & R2 & p-valor \\
\hline \multirow{2}{*}{ Pulgar } & $6-25$ & Hombres & 0,510 & $\mathrm{p}<0,001$ \\
& & Mujeres & 0,231 & $\mathrm{p}<0,001$ \\
& & Todos & 0,325 & $\mathrm{p}<0,001$ \\
\cline { 2 - 5 } & $26-58$ & Hombres & 0,147 & $\mathrm{p}=0,159$ \\
& & Mujeres & 0,028 & $\mathrm{p}=0,539$ \\
& & Todos & 0,051 & $\mathrm{p}=0,221$ \\
\hline Índice & $6-25$ & Hombres & 0,554 & $\mathrm{p}<0,001$ \\
& & Mujeres & 0,264 & $\mathrm{p}<0,001$ \\
& & Todos & 0,357 & $\mathrm{p}<0,001$ \\
\cline { 2 - 5 } & $26-58$ & Hombres & 0,065 & $\mathrm{p}=0,360$ \\
& & Mujeres & 0,123 & $\mathrm{p}=0,183$ \\
& & Todos & 0,053 & $\mathrm{p}=0,214$ \\
\hline
\end{tabular}

Tab. 3. Resultados de las regresiones logarítmicas derivadas de la población de referencia para el pulgar y para el índice.
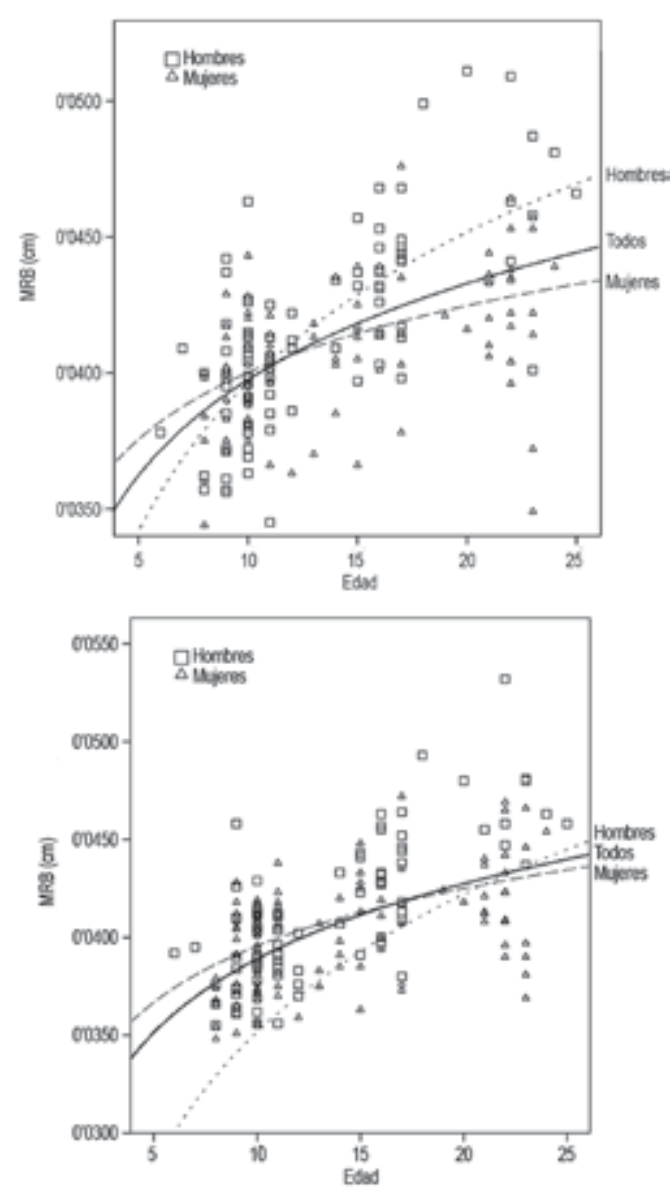

Fig. 7. Población actual de referencia (individuos de 25 y menos años). Gráficos del modelo de regresión logarítmica determinado utilizando la anchura media de la cresta epidérmica (Mean Ridge Breath, MRB) del pulgar (A) y del índice (B). 
de una persona de la población de referencia si las huellas son de individuos infantiles y jóvenes, pero no de mayores de 25 años, ya que no se observa aumento de la anchura de la cresta en la madurez. La medida más pequeña hallada en la cerámica $(0,0557 \mathrm{~cm})$ es mayor que cualquiera de las medidas encontradas en individuos de entre 6 y 25 años. Por ello, es muy probable que las identificadas en la cerámica pertenezcan a un individuo adulto cuya edad exacta no se puede estimar.

\subsection{Estimación del sexo del individuo}

En individuos mayores de 25 años hay diferencias significativas entre sexos en el $M R B$ en pulgar (test de Mann-Whitney: $Z=-2$ '195; $N=31$; $\mathrm{p}<0$ '05) e índice (test de Mann-Whitney: $Z=-$ 2'293; $\mathrm{N}=31 ; \mathrm{p}<0$ ’05), presentando los hombres valores más elevados (Fig. 8). Por ello se han realizado análisis discriminantes para determinar el sexo de las huellas de la cerámica. Usando los datos procedentes de la muestra contemporánea, se ha obtenido una función discriminante para cada dedo: Pulgar: $\mathrm{y}=303^{\prime} 886 * \mathrm{x}-14^{\prime} 045$; Índice: $\mathrm{y}=282^{\prime} 334^{*} \mathrm{x}-13^{\prime} 181$ (donde $x$ corresponde a la anchura de la cresta en $\mathrm{cm}$ ).

La proporción de variabilidad total debida a las diferencias entre grupos queda recogida en el

\begin{tabular}{|c|l|c|c|}
\hline \multicolumn{2}{|l|}{} & Pulgar & Índice \\
\hline A & Lambda de Wilks & 0,848 & 0,811 \\
& p-valor & $<0,05$ & $<0,05$ \\
& Correlación canónica & 0,390 & 0,434 \\
& Punto de corte & 0,0135 & 0,0150 \\
\hline \multirow{2}{*}{ B } & Lambda de Wilks & 0,867 & 0,811 \\
& p-valor & $<0,05$ & $<0,05$ \\
& Correlación canónica & 0,365 & 0,434 \\
& Punto de corte & 0,0125 & 0,0160 \\
\hline
\end{tabular}

Tab. 4. Coeficientes y significación de los dos análisis discriminantes derivados de la población de referencia a partir de los datos de pulgar e índice (A) y de los datos de la zona I de pulgar e índice (B)

valor de correlación canónica. Contrariamente, la lambda de Wilks es la proporción de variabilidad no explicada por el modelo. Es elevada para el pulgar y el índice, pero los p-valores indican que el modelo es útil para hacer clasificaciones. Los valores son similares en los dos dedos que presentan, por tanto, un nivel de distinción sexual similar (Tab. 4A).

Se ha calculado la exactitud de la clasificación sexual en la muestra de población española (Tab. 5) utilizando las funciones discriminantes obtenidas. El 71\% de los individuos se clasifican correctamente, tanto para el dedo pulgar como para el índice, pero en hombres, en una propor-
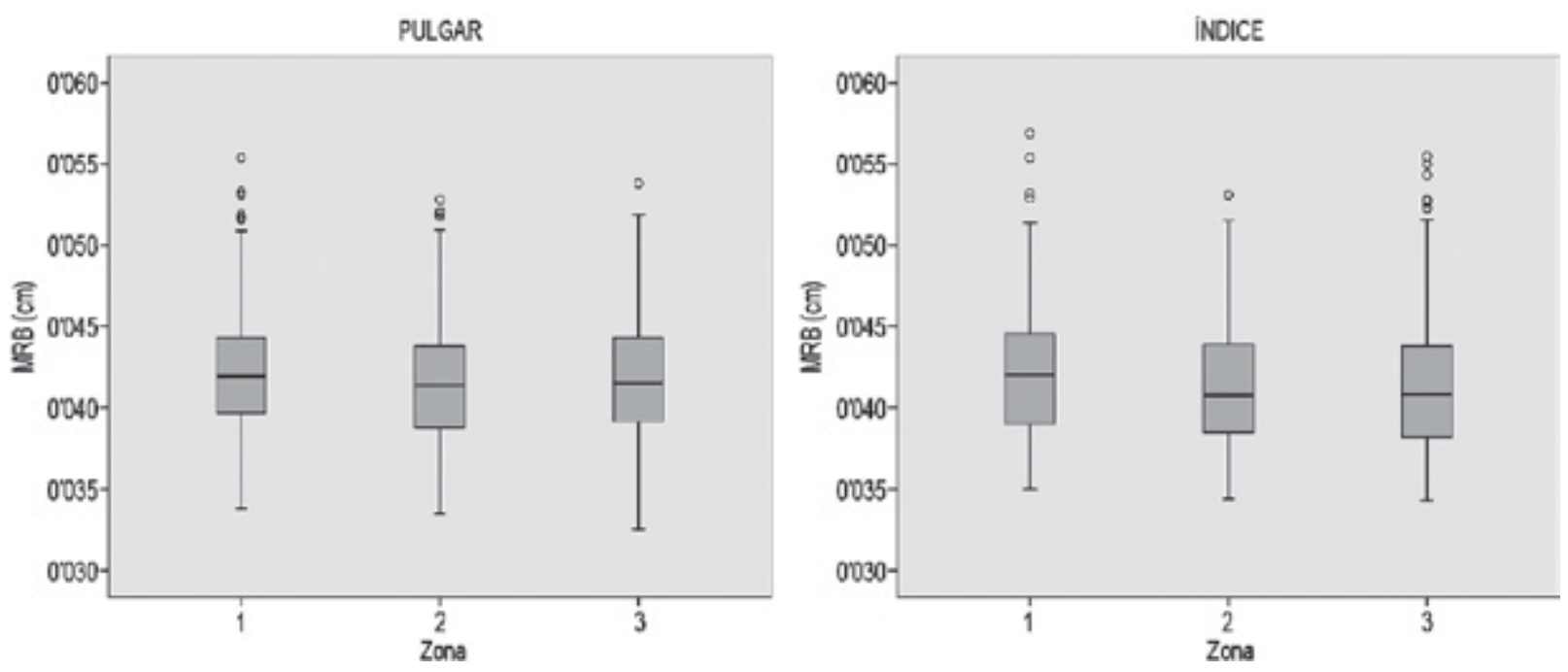

Fig. 8. Población actual de referencia (mayores de 25 años). Diagramas de caja para la anchura media de la cresta epidérmica (Mean Ridge Breath, MRB) de pulgar e índice para cada sexo.

Trab. Prehist., 73, N. ${ }^{\circ}$ 1, enero-junio 2016, pp. 147-159, ISSN: 0082-5638

doi: $10.3989 /$ tp. 2016.12168 


\begin{tabular}{|c|c|c|c|c|c|}
\hline & & Sexo real & Clasi & & Exactitud \\
\hline \multirow[t]{3}{*}{ Pulgar } & & & Hombre (n) & Mujer (n) & \\
\hline & $\begin{array}{l}\text { Validación no } \\
\text { cruzada }\end{array}$ & $\begin{array}{l}\text { Hombre }(n=15) \\
\text { Mujer }(n=16)\end{array}$ & $\begin{array}{l}9 \\
3 \\
\end{array}$ & $\begin{array}{r}6 \\
13 \\
\end{array}$ & $71 \%$ \\
\hline & $\begin{array}{l}\text { Validación cruzada } \\
\text { jackknife }\end{array}$ & $\begin{array}{l}\text { Hombre }(\mathrm{n}=15) \\
\text { Mujer }(\mathrm{n}=16)\end{array}$ & $\begin{array}{l}9 \\
3\end{array}$ & $\begin{array}{r}6 \\
13 \\
\end{array}$ & $71 \%$ \\
\hline \multirow[t]{3}{*}{ Índice } & & & Hombre (n) & Mujer (n) & \\
\hline & $\begin{array}{l}\text { Validación no } \\
\text { cruzada }\end{array}$ & $\begin{array}{l}\text { Hombre }(n=15) \\
\text { Mujer }(n=16)\end{array}$ & $\begin{array}{r}10 \\
4\end{array}$ & $\begin{array}{r}5 \\
12\end{array}$ & $71 \%$ \\
\hline & $\begin{array}{l}\text { Validación cruzada } \\
\text { jackknife }\end{array}$ & $\begin{array}{l}\text { Hombre }(n=15) \\
\text { Mujer }(n=16)\end{array}$ & $\begin{array}{r}10 \\
4\end{array}$ & $\begin{array}{r}5 \\
12\end{array}$ & $71 \%$ \\
\hline
\end{tabular}

Tab. 5. Resultados y exactitud de la clasificación de la muestra de la población de referencia usando la función discriminante para el pulgar y para el índice.

ción menor que en mujeres ( $60 \%$ vs. $81,15 \%$ en pulgar y $66,67 \%$ vs. $75 \%$ en índice) (Tab. 5). La validación cruzada jackknife, donde cada caso se vuelve a clasificar según una nueva función discriminante calculada sin tenerlo en cuenta, da los mismos valores de exactitud que la validación no cruzada.

Estas funciones se han usado para hacer la estimación del sexo de las huellas encontradas en la cerámica. A partir de las medidas de la cerámica, se obtiene un valor de $y$ concreto que permite clasificar la medida en el grupo hombre o mujer, según un punto de corte que también aporta el modelo (Tab. 4A). En este caso, todas las medidas obtenidas en la cerámica han sido clasificadas en el grupo de hombres con más de un $91 \%$ de probabilidad, utilizando la función discriminante correspondiente a ambos dedos (Tab. 6).

\begin{tabular}{|l|c|c|c|c|}
\hline & \multicolumn{2}{|c|}{ Pulgar } & \multicolumn{2}{c|}{ Índice } \\
\hline & Grupo & Probabilidad & Grupo & Probabilidad \\
\hline Región 1 & Hombre & 92,610 & Hombre & 92,727 \\
\hline Región 2 & Hombre & 92,945 & Hombre & 93,074 \\
\hline Región 3 & Hombre & 99,162 & Hombre & 99,273 \\
\hline Región 4 & Hombre & 91,323 & Hombre & 91,380 \\
\hline Región 5 & Hombre & 92,438 & Hombre & 92,547 \\
\hline
\end{tabular}

Tab. 6. Resultado de la clasificación sexual de las huellas de la cerámica de la Edad del Bronce de La Canal dels Avellaners realizada a partir de las funciones discriminantes obtenidas con las medidas medias de cada dedo.
Para aportar más robustez a los resultados y dado el elevado valor de la anchura de las huellas encontradas en la cerámica, se efectuó otro análisis discriminante para cada uno de los dedos, utilizando las medidas de la zona I en las huellas de la población de referencia, ya que son las que muestran el valor más alto de todas las zonas. Las funciones discriminantes obtenidas con estos datos son los siguientes: Pulgar: $\mathrm{y}=285^{\prime} 028 * \mathrm{x}-$ 13'352; Índice: $\mathrm{y}=258^{\prime} 260 * \mathrm{x}-12^{\prime} 070$ (donde $x$ corresponde a la anchura de la cresta en $\mathrm{cm}$ ).

Los valores de la lambda de Wilks y de la correlación canónica son similares a los obtenidos empleando las medias de las medidas de cada dedo (Tab. 4B). Los p-valores en este caso también indican que los modelos son útiles para la clasificación.

La exactitud resultante con validación no cruzada y validación cruzada jackknife es de 64,5\% para el pulgar (Tab. 7). En el índice, la clasificación correcta se produce en un 79,3\% de los casos mediante validación no cruzada y en un $75,9 \%$ mediante validación cruzada jackknife (Tab. 7). La exactitud es menor en hombres que en mujeres $(60 \%$ vs. $68,75 \%$ en pulgar por cualquier validación; $71,43 \%$ vs. $86,67 \%$ en índice con validación no cruzada y $64,29 \%$ vs. $86,67 \%$ en índice con validación cruzada), tal como ocurría considerando la media de las medidas en cada dedo. Nuevamente, las medidas obtenidas en la cerámica se han clasificado siguiendo el modelo dentro del grupo de hombres con más de un $87 \%$ de probabilidad en todos los casos (Tab. 8). 


\begin{tabular}{|c|c|c|c|c|c|}
\hline & & Sexo real & Clasifi & & Exactitud \\
\hline \multirow[t]{3}{*}{ Pulgar } & & & Hombre (n) & Mujer (n) & \\
\hline & $\begin{array}{l}\text { Validación no } \\
\text { cruzada }\end{array}$ & $\begin{array}{l}\text { Hombre }(n=15) \\
\text { Mujer }(n=16)\end{array}$ & $\begin{array}{l}9 \\
5\end{array}$ & $\begin{array}{r}6 \\
11\end{array}$ & $64,5 \%$ \\
\hline & $\begin{array}{l}\text { Validación cruzada } \\
\text { jackknife }\end{array}$ & $\begin{array}{l}\text { Hombre }(n=15) \\
\text { Mujer }(n=16)\end{array}$ & $\begin{array}{l}9 \\
5 \\
\end{array}$ & $\begin{array}{r}6 \\
11 \\
\end{array}$ & $64,5 \%$ \\
\hline \multirow[t]{3}{*}{ Índice } & & & Hombre (n) & Mujer (n) & \\
\hline & $\begin{array}{l}\text { Validación no } \\
\text { cruzada }\end{array}$ & $\begin{array}{l}\text { Hombre }(n=14) \\
\text { Mujer }(n=15)\end{array}$ & $\begin{array}{r}10 \\
2 \\
\end{array}$ & $\begin{array}{r}4 \\
13 \\
\end{array}$ & $79,3 \%$ \\
\hline & $\begin{array}{l}\text { Validación cruzada } \\
\text { jackknife }\end{array}$ & $\begin{array}{l}\text { Hombre }(n=14) \\
\text { Mujer }(n=15)\end{array}$ & $\begin{array}{l}9 \\
2\end{array}$ & $\begin{array}{r}5 \\
13\end{array}$ & $75,9 \%$ \\
\hline
\end{tabular}

Tab. 7. Resultados y exactitud de la clasificación de la muestra de la población de referencia usando la función discriminante para la zona I del pulgar y para la zona I del índice.

\begin{tabular}{|l|c|c|c|c|}
\hline & \multicolumn{2}{|c|}{ Pulgar } & \multicolumn{2}{|c|}{ Índice } \\
\hline & Grupo & Probabilidad & Grupo & Probabilidad \\
\hline Región 1 & Hombre & 88,680 & Hombre & 90,971 \\
\cline { 1 - 1 } Región 2 & Hombre & 89,107 & Hombre & 91,359 \\
\cline { 1 - 1 } Región 3 & Hombre & 98,212 & Hombre & 98,874 \\
\cline { 1 - 1 } Región 4 & Hombre & 87,067 & Hombre & 89,490 \\
\cline { 1 - 1 } Región 5 & Hombre & 88,460 & Hombre & 90,772 \\
\hline
\end{tabular}

Tab. 8. Resultado de la clasificación sexual de las huellas de la cerámica de la Edad del Bronce de La Canal dels Avellaners realizada a partir de las funciones discriminantes obtenidas con las medidas de la zona I de cada dedo.

\section{DISCUSIÓN}

Los resultados de este estudio concuerdan con investigaciones previas que indican que la anchura de las crestas epidérmicas puede ser utilizada para inferir la edad aproximada y el sexo de individuos que dejaron sus huellas en cerámicas antiguas (Kamp et al. 1999; Králík et al. 2002; Králík y Novotný 2003). Estos estudios permiten hacer buenas estimaciones pero, normalmente, implican una cierta dificultad, dado que las huellas antiguas son fragmentarias y pequeñas. El hecho de encontrar pocos paleodermatoglifos además de parciales, en una misma cerámica puede suponer un problema. El $M R B$ varía considerablemente entre las regiones de la huella de un individuo e incluso entre las zonas o secciones de una región particular (Králík y Novotný 2003), de manera que un conjunto con huellas pequeñas y poco representativas no es una buena muestra de análisis.

Además, el proceso de fabricación de la cerámica puede distorsionar las crestas. $\mathrm{El}$ riesgo de error aumenta si solo hay una huella antigua (Králík et al. 2002). En la cerámica de La Canal dels Avellaners este problema no se da, pues hay varias. No obstante, se podría haber producido una distorsión en la huella de la región 3 , donde las medidas superan bastante las de las otras regiones. La distorsión podría haber sido fruto de un contacto entre dedo y cerámica con diferente intensidad. Králík y Novotný (2003) defienden que las huellas dejadas en las cerámicas difieren según las acciones que las originen: tocar $\mathrm{y}$ sujetar no es como modelar con las puntas o los márgenes de los dedos. Otra explicación de este hecho podría ser que esta huella concreta perteneciera a otra región del mismo dedo, a otro dedo, a la palma de la mano o bien a otro individuo con mayor anchura de las crestas. No obstante, la variabilidad intrapersonal es poco probable, ya que la diferencia entre la región 3 y el resto es muy marcada. Así mismo, la variabilidad interpersonal tampoco es muy probable: las medidas de esta huella son excesivamente grandes y ni siquiera comparables a las de ningún individuo de la población actual. Además, en cerámicas y otros objetos antiguos, algunas regiones dermatoglíficas están preferentemente representadas (Kamp et al. 1999). La homogeneidad en los valores del $M R B$ se debe al uso de los mismos dedos y de regiones 
concretas de la palma en el proceso de modelaje (Králík y Novotný 2003). Ninguna explicación es descartable por completo pero la distorsión de la huella es la más probable.

La estimación de la edad y el sexo en las huellas antiguas encontradas se basa en dos supuestos básicos. El primero es aceptar un valor concreto para la contracción del material cerámico durante el proceso de cocción. En la cerámica de La Canal dels Avellaners, se ha utilizado una corrección del $7,5 \%$ por sus buenos resultados en estudios previos (Králík y Novotný 2003; Králík, comunicación personal). Los valores de las medidas de las huellas de esta cerámica eran tan elevados que, incluso sin corrección, se habrían clasificado en el grupo de hombres. Gracias a esta característica particular se ha descartado esta fuente de error existente en otros casos. No obstante el grado de contracción se debería determinar en cada material cerámico particular. El segundo supuesto consiste en aceptar que el $M R B$ en la población de la Edad del Bronce es el mismo que se da en la población española actual. La variabilidad en las huellas tiene un componente poblacional. Jantz y Parham (1978) estudiaron la anchura de las crestas en estudiantes Yoruba (Nigeria), ingleses y judíos, demostrando diferencias sustanciales en ambos sexos. También se ha observado que la anchura de la cresta covaría con otras características dermatoglíficas. En esta investigación se ha intentado minimizar esta variabilidad, comparando las huellas antiguas con individuos de la población actual de la misma región geográfica (España). No obstante, los resultados de algunos estudios recientes muestran que los métodos de predicción de sexo mediante huellas tienen un éxito parcial cuando la muestra problema es de la misma población que la de referencia, éxito que se reduce con poblaciones no relacionadas (Galeta et al. 2014). Este problema queda minimizado en la cerámica estudiada gracias a los valores muy altos del $M R B$ en sus huellas. La anchura de las crestas está relacionada con la medida de la palma, la longitud de los metacarpos y la medida del esqueleto en general (Babler 1990; Loesch y Lafranchi 1990). Algunas investigaciones han evidenciado la tendencia a un aumento generacional de la altura en las poblaciones (aumento secular de la estatura) por la mejora en la alimentación y en las condiciones ambientales en general (Valls 1980; Subirà y Malgosa 1988). Por tanto, en la población de la Edad de Bronce, los individuos muy probablemente serían más pequeños (Angel 1984; Hermanussen 2003) y sus huellas dactilares tendrían una menor anchura que en los individuos actuales, considerando que pertenecen a la misma población. Si comparásemos las huellas encontradas en la cerámica con las de la población de la Edad de Bronce, seguramente las clasificaríamos como masculinas con una mayor probabilidad que la obtenida en la comparación con población actual.

No se ha podido determinar a qué dedo concreto pertenecen las huellas que se encuentran en la cerámica. En este estudio, el $M R B$ del dedo índice en adultos es ligeramente superior al de pulgar, pero esto se debe, muy probablemente, al tamaño de muestra. No obstante, en estudios previos con población caucásica española, tanto en hombres como en mujeres, el pulgar y el índice muestran una menor densidad de las crestas (y, por tanto, una mayor anchura) en las zonas de análisis, siendo el pulgar el de mayor anchura de cresta (Gutiérrez-Redomero et al. 2008). Dada la gran anchura de las huellas reconocidas, lo más posible es que los paleodermatoglifos pertenecieran a uno de estos dedos, probablemente al pulgar. Tampoco se puede descartar una huella palmar, ya que investigaciones previas observan que la anchura media de cresta para hombres de población caucásica española es de $0,0520 \mathrm{~cm}$ (Gutiérrez-Redomero y Alonso-Rodríguez 2013), mientras la anchura menor encontrada en este estudio de $0,0557 \mathrm{~cm}$.

Muchos casos de estudio de paleodermatoglifos han determinado que las dimensiones de las huellas sobre cerámicas y otros artefactos entraban en el rango de variación de mujeres o niños (Králík et al. 2002; Králík y Einwögerer 2010). La frecuencia de huellas, sobre todo de adolescentes, al demostrar su participación en este tipo de tareas, ha repercutido en la interpretación del arte paleolítico en general (Králík y Einwögerer 2010) y en la creación de las cerámicas antiguas en particular. Durante la Edad de Bronce, se han demostrado colaboraciones entre niños y adultos (Jägerbrand 2007). Sin descartar por completo otras hipótesis, las evidencias sugieren que las huellas reconocidas en la cerámica de La Canal dels Avellaners pertenecen a un único individuo, muy probablemente un hombre adulto. Sin embargo, no se puede asegurar que las huellas encontradas sean del alfarero y no de una persona que tocó la cerámica durante el proceso de manufactura. 
La mayoría de las huellas dactilares presentes en objetos antiguos pasan desapercibidas, a pesar de que su estudio puede aportar mucha información sobre la persona que los fabricó o en última instancia, que tuvo contacto con ellos. Las determinaciones de este tipo son de gran importancia no sólo en la investigación arqueológica, sino también en la forense, ya que es posible determinar el género, la edad aproximada e incluso la etnia de un individuo a partir de huellas de origen desconocido.

\section{AGRADECIMIENTOS}

El Museo Comarcal de Berga cedió la pieza y la Unidad de Inspección Ocular de los Mossos d'Esquadra las imágenes de la cerámica. Miroslav Králík nos asesoró. También agradecemos la colaboración de Annick Labeeuw del Centro de Regulación Genómica de Barcelona y la participación en el proyecto de la Escola Pia de Caldes, la Escola Pia de Terrassa, la Escola Joan Roca de Barcelona, la Escola Sol i Lluna de Castellar del Vallès, el Institut Barri Besós del Barcelona y todos los demás voluntarios.

\section{BIBLIOGRAFÍA}

Aase, J. y Lyons, R. B. 1971: "Technique for recording dermatoglyphics". Lancet 1: 432-433.

Acree, M. A. 1999: "Is there a gender difference in fingerprint ridge density?". Forensic Science International 102: 35-44.

Alarcón García, E. 2015: "Social relations between adulthood and childhood in the Early Bronze Age site of Peñalosa (Baños de la Encina, Jaen, Spain)". En M. Sánchez Romero, E. Alarcón García, G. Aranda Jiménez (eds.): Children, spaces and identity. Oxbow Books. Oxford: 59-74.

Almécija, S. 2009: "Evolution of the hand in Miocene apes: implications for the appearance of the human hand". Tesis Doctoral, Universitat Autònoma de Barcelona. http:/www.tdx.cat/bitstream/handle/10803/3707/ (consulta 15-IV-2016).

Angel, J. M. 1984: "Health as a crucial factor in the changes from hunting to developed farming in the Eastern Mediterranean". En M. N. Cohen y G. J. Armelagos (eds.): Paleopathology at the origins of agriculture. Academic Press. New York: 51-73.
Babler, W. 1990: "Prenatal communalities in epidermal ridge development”. En N. M. Durham y C. C. Plato (eds.): Trends in Dermatoglyphic Research. Kluwer Academic Publishers. Dordrecht: 54-68.

Carreras, J. 1990: “La canal dels Avellaners". En J. Castany, E. Sánchez, L. A. Guerrero, J. Carreras, R. Mora y G. Vila (eds.): El Berguedà: de la Prehistòria a l'antiguitat. Ámbit de Recerques del Berguedà i Ajuntament de Puig-Reig. Berga: 29-76.

Cummins, H. 1941: "Ancient fingerprints in clay". The Scientific Monthly 52: 389-402.

Cummins, H.; Waits, J. W. y McQuitty, J. T. 1941: “The breaths of epidermal ridges on the finger tips and palms: a study of variation". American Journal of Anatomy 68: 127-150.

David, T. J. 1981: "Distribution, age, and sex variation of the mean epidermal ridge breadth". Human Heredity 31: 279-282.

Eshak, G. A.; Zaher, J. F.; Hasan, E. I. y Ewis, A. A. E. 2012: "Sex identification from fingertip features in Egyptian population". Journal of Forensic and Legal Medicine 20: 46-50.

Galeta, P.; Jaroslav, B. y Lázničková-Galetová, M. 2014: "Is sex estimation from handprints in prehistoric cave art reliable? A view from biological and forensic anthropology". Journal of Archaeological Science 45: 141-149.

Gutiérrez-Redomero, E.; Alonso, M. C. y Dipierri, J. E. 2011: "Sex differences in fingerprint ridge density in the Mataco-Mataguayo population". Journal of Comparative Human Biology 62: 487-499.

Gutiérrez-Redomero, E.; Alonso, M. C.; Romero, E. y Galera, V. 2008: "Variability of fingerprint ridge density in a sample of Spanish Caucasians and its application to sex determination". Forensic Science International 180: 17-22.

Gutiérrez-Redomero, E.; Quirós, J. A.; Rivaldería, N. y Alonso, M. C. 2013a: "Topological variability of fingerprint ridge density in a sub-Saharan population sample for application in personal identification". Journal of Forensic Sciences 58: 592-600.

Gutiérrez-Redomero, E.; Sánchez-Andrés, A.; Rivaldería, N.; Alonso-Rodríguez, C.; Dipierri, J. E. y Martín L. M. 2013b: "A comparative study of topological and sex differences in fingerprint ridge density in Argentinean and Spanish population samples". Journal of Forensic and Legal Medicine 20: 419-429.

Hermanussen, M. 2003: "Stature of early Europeans". Hormones 2 (3): 175-178.

Holt, S. B. 1968: The genetics of dermal ridges. Charles C. Thomas. Springfield.

IBM SPSS Statistics 21. 2012: Software for windows. IBM Corporation. Armonk.

ImageJ 1.45s. 2011: Public domain software for Windows. Wayne Resberd, National Institutes of Health. Bethesda. 
Jantz, R. L. y Parham, K. R. 1978: "Racial differences in dermal ridge breadth". Human Biology 50: 33-40.

Jägerbrand, M. 2007: "Why the world needs another journal". Journal of Ancient Fingerprints 1: 1.

Kamp, K. A.; Timmerman, N.; Lind, G.; Graybill, J. y Natowsky I. 1999: "Discovering childhood: using fingerprints to find children in the archaeological record". American Antiquity 64: 309-315.

Králík, M. y Einwögerer, T. 2010: "Imprints discovered on Paleolithic ceramics form the Krems-Wachtberg and Krems-Hundssteig sites, lower Austria". En C. Neugebauer- Maresch, L. Owen (eds.): New aspects of the Central and Eastern European Upper Palaeolithic - Methods, Chronology, Technology and Subsistences. Österreichische Akademie der Wissenschaften. Wien: 255-272.

Králík, M. y Novotoný, V. 2003: “Epidermal ridge breadth: an indicator of age and sex in paleodermatoglyphics". Variability and Evolution 11: 5-30.

Králík, M.; Novotoný, V. y Oliva, M. 2002: "Fingerprint on the Venus of Dolní Vêstonice I'. Anthropologie 40: 107-113.

Loesch D. Z. 1983: Quantitative Dermatoglyphics: classification, genetics and pathology. Oxford University Press. New York.

Loesch, D. Z. y Lafranchi, M. 1990: "Relationships of epidermal ridge patterns with body measurements and their possible evolutionary significance". American Journal of Physical Anthropology 82: 183-189.

McGrew, W. C. y Marchant, L. F. 1994: "Primate etology: a perspective on human and nonhuman handedness". En P. Bock (ed.): Handbook of Psychological Anthropology. Greewood Press. Westport: 171-184.

Mundorff, A. Z.; Bartelink, E. J. y Murad, T. A. 2014: "Sexual dimorphism in finger ridge breath measurements: a tool for sex estimation from fingerprints". Journal of Forensic Science 59: 891-897.
Nayak, V. C.; Rastogi, P.; Kanchan, T.; Lobo, S. W.; Yoganarasimha, K.; Nayak, S.; Rao, N. G; Pradeep Kumar, G.; Suresh Kumar Shetty, B. y Menezes, R. G. 2010a: "Sex differences from fingerprint ridge density in the Indian population". Journal of Forensic and Legal Medicine 17: 84-86.

Nayak, V. C.; Rastogi, P.; Kanchan, T.; Yoganarasimha, K.; Kumar, G.P. y Menezes, R.G. 2010b: "Sex differences from fingerprint ridge density in Chinese and Malaysian populations". Forensic Science International 197: 67-69.

Ohler, E. A y Cummins, H. 1942: "Sexual differences in breadths of epidermal ridges on finger tips and palms". American Journal of Physical Anthropology 29: 341-362.

Penrose, L. S. 1968: "Memorandum on dermatoglyphic nomenclature". Birth defects: original article series 4: 1-13.

Perelle, I. B. y Ehrman, L. 1994: "An international study of human handedness: the data". Behavior Genetics 24: 217-227.

Rivaldería, N.; Sánchez-Andrés, A.; Alonso-Rodríguez, C.; Dipierri, J. E. y Gutiérrez-Redomero, E. 2016: "Fingerprint ridge density in the Argentinean population and its application to sex inference: a comparative study". HOMO - Journal of Comparative Human Biology 67: 65-84

Subirá, M. E. y Malgosa, A. 1988: "Somatometric study of the hands". International Journal of Anthropology 3: 329-338.

Taduran, R. J. O.; Tadeo, A. K. V.; Escalona, N. A. C. y Townsend G. C. 2015: "Sex determination from fingerprint ridge density and white line counts in Filipinos". HOMO - Journal of Comparative Human Biology. DOI: 10.1016/j.jchb.2015.11.001.

Valls, A. 1980: Introducción a la Antropología: fundamentos de la evolución y de la variabilidad biológica del hombre. Labor Universitaria, Manuales. Barcelona. 